\title{
Incidence of corneal melting in association with systemic disease in the Yorkshire Region, 1995-7
}

\author{
M McKibbin, J D Isaacs, A J Morrell
}

\begin{abstract}
Aims-To estimate the incidence of corneal melting or necrotising keratitis in association with systemic disease in the Yorkshire Region and to determine the type and duration of the systemic association.

Methods-In a prospective study, vigorous attempts were made to identify all patients presenting with newly diagnosed corneal melting over a 3 year period.

Results-27 patients were identified during the study period. Rheumatoid arthritis and Wegener's granulomatosis were the most common disease associations. Corneal melting was a late complication of rheumatoid arthritis, but usually occurred during early and overt systemic disease in patients with Wegener's granulomatosis.

Conclusion-The annual incidence of corneal melting in the Yorkshire Region is $3.01 / \mathrm{million} /$ year $(95 \% \mathrm{CI}=0.7-9.6)$.

(Br f Ophthalmol 1999;83:941-943)
\end{abstract}

Corneal ulceration with rapidly progressive stromal thinning or melting occurs most commonly in association with a systemic vasculitis. ${ }^{1}$ These changes may occur either in the peripheral cornea, where they may be accompanied by adjacent scleritis, or else in the central or paracentral cornea. ${ }^{2} 3$ The process of ulceration and melting may be initiated by a variety of factors including local, immune mediated vasculitis, and ocular surface disease, especially keratoconjunctivitis sicca and corneal infection. Without appropriate treatment, progressive corneal thinning with perforation and loss of the globe may occur.

The diagnosis of corneal melting or necrotising keratitis is largely based on the clinical appearance of the eye, especially in the context of systemic vasculitis. ${ }^{14}$ Though seldom necessary, support for this clinical impression may be obtained from laboratory investigation, anterior segment angiography, and histological evidence of local or systemic vasculitis when available. $^{125}$ The disease may be subdivided initially by anatomical site into central and peripheral forms, or by the likely pathogenesis, into inflammatory and non-inflammatory forms based on the relative contribution of vasculitis and ocular surface disease respectively. ${ }^{4}$ Features of inflammatory vasculitis include limbal inflammation, perilesional infiltrate, and well defined ulcers with sharp edges, whereas the non-inflammatory ulcers have flatter edges with minimal or no limbal inflammation. ${ }^{4}$ A tendency exists for the inflammatory ulceration to occur in the peripheral cornea and for the noninflammatory ulceration to occur in the central or paracentral cornea. ${ }^{2-4}$ However, neither of these inflammatory and anatomical classifications is absolute and considerable overlap exists between the groups. ${ }^{46}$

The incidence of corneal melting in association with systemic disease is unknown. The aim of this study was to determine the incidence of corneal melting in a well defined population and the type and duration of the systemic disease association.

\section{Method}

For the period between January 1995 and December 1997, we attempted to identify all patients presenting with a new diagnosis of peripheral or central corneal melting in association with a systemic disease within the Yorkshire Region.

Patients were identified through several sources:

(1) Letters were sent to practising consultant ophthalmologists and rheumatologists working in the Yorkshire Region at intervals of approximately 6 months between June 1995 and January 1998 asking for the details of affected patients.

(2) The computerised records of the histopathology departments of the two teaching hospitals in the region were searched for a histological or clinical diagnosis of corneal melting in association with a systemic vasculitis.

(3) Some patients were encountered during the clinical rotation of one of the authors $(\mathrm{MM})$ as an ophthalmologist in training.

After identification, the case notes of all patients were reviewed in order to determine the anatomical site of the melting process and the type and duration of the associated systemic disease. Patients were included only if a new diagnosis of corneal melting in a previously unaffected eye was made during the study period. Patients having continued treatment for presentation before the study period were excluded.

The denominator population was based on the mid year estimate for 1995 of the seven district health authorities in the Yorkshire Region as provided by the Office of National Statistics. ${ }^{7}$ These are the most recent data for the area. As all of the patients identified were adult, we have used the adult population figures ( $>14$ years) in this study.

Ninety five per cent confidence intervals (CI) were calculated for the observed number of cases. ${ }^{8}$
Accepted for publication 29 March 1999 
Table 1 Age, sex, site of melting, and systemic disease association for the 27 patients included in the study

\begin{tabular}{|c|c|c|c|c|c|}
\hline Year & Sex & $\begin{array}{l}\text { Age at } \\
\text { presentation }\end{array}$ & Site of melting & $\begin{array}{l}\text { Systemic disease } \\
\text { association }\end{array}$ & $\begin{array}{l}\text { Duration of associated } \\
\text { disease in years }\end{array}$ \\
\hline \multirow[t]{9}{*}{1995} & M & 55 & Peripheral & RA & 14 \\
\hline & $\mathrm{F}$ & 73 & Central & RA & 15 \\
\hline & $\mathrm{F}$ & 68 & Peripheral & RA & 24 \\
\hline & $M$ & 80 & Central & RA & 13 \\
\hline & M & 68 & Peripheral & RA & 29 \\
\hline & $M$ & 61 & Peripheral & RA & 30 \\
\hline & $\mathrm{F}$ & 75 & Peripheral & RA & 39 \\
\hline & $\mathrm{F}$ & 71 & Central & RA & 3 \\
\hline & $\mathrm{F}$ & 69 & Central & RA & 14 \\
\hline \multirow[t]{11}{*}{1996} & $\mathrm{~F}$ & 77 & Peripheral & RA & 20 \\
\hline & $\mathrm{F}$ & 72 & Peripheral & RA & 35 \\
\hline & $\mathrm{F}$ & 77 & Peripheral & RA & 24 \\
\hline & $\mathrm{F}$ & 74 & Peripheral & WG & 1 \\
\hline & $M$ & 50 & Peripheral & RA & 30 \\
\hline & $\mathrm{F}$ & 74 & Peripheral & RA & 8 \\
\hline & M & 72 & Peripheral & RA & 26 \\
\hline & $M$ & 77 & Peripheral & RA & 16 \\
\hline & $M$ & 26 & Peripheral & WG & 0.5 \\
\hline & $\mathrm{F}$ & 71 & Peripheral & RA & 13 \\
\hline & $M$ & 70 & Central & RA & 12 \\
\hline \multirow[t]{7}{*}{1997} & $\mathrm{~F}$ & 71 & Peripheral & WG & 0 \\
\hline & $\mathrm{M}$ & 71 & Peripheral & MP & 0 \\
\hline & M & 68 & Peripheral & RA & 20 \\
\hline & $\mathrm{F}$ & 76 & Central & RA & 15 \\
\hline & M & 40 & Peripheral & WG & 2 \\
\hline & $\mathrm{F}$ & 63 & Peripheral & RA & 12 \\
\hline & $M$ & 67 & Peripheral & WG & 0 \\
\hline
\end{tabular}

$\mathrm{RA}=$ rheumatoid arthritis, $\mathrm{WG}=$ Wegener's granulomatosis, $\mathrm{MP}=$ microscopic polyarteritis.

\section{Results}

Thirty three patients with a diagnosis of systemic disease associated corneal melting were identified, of whom six had originally presented with corneal melting outside of the study period and were excluded. Twenty seven were then included in the study, eight of whom were identified through more than one individual or source. Of the 27 , the average age at the time of corneal melting was 67.2 years and 14 were female.

Rheumatoid arthritis was the most commonly associated systemic disease in 21 patients, followed by Wegener's granulomatosis in five cases. A single case was associated with microscopic polyarteritis. For those patients with rheumatoid arthritis, the corneal melting was a late feature occurring 19.6 years after diagnosis on average. In comparison, corneal disease was present at the time of original presentation in three of the six cases with Wegener's granulomatosis or microscopic polyarteritis and after 6 months on average. These data are shown in Table 1.

For 1995, the estimated adult (>14 years) population for the Yorkshire Region was 2993600 . For the period 1995-7, the annual incidence of corneal melting in association with systemic disease in the Yorkshire Region was therefore $3.01 /$ million/year $(95 \%$ CI $=$ 0-9.6).

\section{Discussion}

The results of this study provide an estimate of the incidence of corneal melting in association with systemic disease for a well defined population. Although direct comparison is not possible, the figure of $3.01 / \mathrm{million} /$ year is in keeping with other UK studies relating to the incidence of the observed disease associations. ${ }^{9}{ }^{10}$ In these, the overall incidence of rheumatoid vasculitis and Wegener's granulomatosis is reported as 12.5 and $8.5 /$ million/ year respectively. Ophthalmic complications of all types occur in 16-28\% of such patients but the specific complication of corneal melting is likely to be much less frequent. ${ }^{911} 12$

Over the 3 year period of the study we attempted to identify all new cases of corneal melting in association with systemic disease. While it is possible that some cases may have been missed, we believe that this is unlikely. Corneal melting is usually painful and visual loss occurs quickly and so early referral to an ophthalmologist is the rule. As the condition is uncommon and requires long term medical and surgical therapy, most patients are managed either locally by consultants with a subspecialty interest in anterior segment disease or are referred to such consultants in tertiary centres. Regular contact with the practising consultants in the region, particularly those with relevant subspecialty interest, would be expected to identify the majority of patients. In this study, all except one of the patients were identified either by communication from practising ophthalmologists or while one of us $(\mathrm{MM})$ was working in a tertiary centre. Eight patients were identified through more than one individual or source.

We have chosen to define the type of melting by the anatomical site. As the case notes were reviewed retrospectively it was rarely possible to determine the inflammatory component to each case. Classification into central or paracentral and peripheral forms was the most readily identifiable method. We have included both the central and peripheral forms of melting under the label of systemic disease associated corneal melting. The distinction into inflammatory and non-inflammatory types, or into vasculitic or surface disease associated, is far from absolute. ${ }^{46}$ All of the patients in this study had an underlying systemic vasculitis and 13 of the 21 patients with rheumatoid arthritis had a history of ocular surface disease, most commonly keratoconjunctivitis sicca. All of the patients with Wegener's granulomatosis or microscopic polyarteritis had peripheral melting and no history of ocular surface disease. For the rheumatoid arthritis patients, peripheral melting was not associated with surface disease but there was a trend for central melting to occur in association with ocular surface disease. However, in our experience, even when cases of central corneal melting in these patients appear to be entirely the result of ocular surface disease, they behave very differently from other cases with an identical ocular surface but no systemic vasculitis. In the former, the inflammatory response to corneal drying, epithelial instability or infection appears to be more rapid and marked. This may be a reflection of an inherent abnormality of immune regulation in these patients.

The corneal disease was often an initial feature in those patients with either Wegener's granulomatosis or microscopic polyarteritis, occurring at a time of overt systemic disease. If the serum ANCA is absent in the early stages of the disease, the corneal signs may contribute to the diagnosis. By contrast, the melting tended to occur as a late feature in those with 
rheumatoid arthritis, some 20 years after diagnosis on average. In our experience, these patients often do not have many clinical or biochemical features of systemic vasculitis at the time of melting, although much of this may be subclinical. ${ }^{13}$ However, many have other features associated with extra-articular vasculitis, such as rheumatoid nodules, positive rheumatoid factor and positive HLA-DR4.

All but one of the cases in this study were associated with either rheumatoid arthritis or Wegener's granulomatosis. The management of these conditions, especially rheumatoid arthritis, has evolved over the past few years, with emphasis now placed on treatment with disease modifying agents in the early course of the disease to prevent the late complications. ${ }^{1415}$ As corneal melting was usually seen as a late feature of rheumatoid arthritis in particular, it may be expected that the incidence of this condition in association with systemic vasculitis will decline in the future. However, increasing clinical awareness and more reliable laboratory diagnosis of both conditions may make such observed trends difficult to ascertain. ${ }^{16}$

In summary, we present the first estimate of systemic disease associated corneal melting in a well defined geographic population. While the most common disease association was rheumatoid arthritis, other cases were seen in the context of ANCA associated disease.
1 Tauber J, de la Maza MS, Hoang-Xuan T, et al. An analysis of therapeutic decision making regarding immunosuppressive chemotherapy for peripheral ulcerative keratitis. Cornea 1990;91:66-73.

2 Watson PG. Vascular changes in peripheral corneal destructive disease. Eye 1990;4:65-73.

3 Kervick GN, Pflugfelder SC, Haimovici R, et al. Paracentral rheumatoid corneal ulceration: clinical features and cyclosporine therapy. Ophthalmology 1992;99:80-8.

4 Bernauer WG, Ficker LA, Watson PG, et al. The management of corneal perforations associated with rheumatoid arthritis. Ophthalmology 1995;102:1325-37.

5 Messmer EM, Foster CS. Destructive corneal and scleral Messmer EM, Foster CS. Destructive corneal and scleral
disease associated with rheumatoid arthritis. Medical and disease associated with rheumatoid arthritis. M.
surgical management. Cornea 1995;14:408-17.

6 Pfister RR, Murphy GE. Corneal ulceration and perforation associated with Sjögren's syndrome. Arch Ophthalmol 1980;98:89-94

7 Population in administrative areas. 1995 Key population and vital statistics - local and health authority areas. Series VS no 22, PP1, no 18. London: Stationery Office, 1998.

8 Clarke GM, Cooke D. Confidence intervals for proportions. Basic course in statistics. London: Edward Arnold, 1986.

9 Watts RA, Carruthers DM, Symmons DP, et al. The incidence of rheumatoid vasculitis in the Norwich Health Authority. Br F Rheum 1994;33:832-3.

10 Carruthers DM, Watts RA, Symmons DPM, et al. Wegener's granulomatosis - first population based estimate of incidence. Br F Rheum 1994;33 (Suppl 2):13.

11 Scott DG, Bacon PA, Tribe CR. Systemic rheumatoid vasculitis: a clinical and laboratory study of 50 cases. Medicine 1981;60:288-97.

12 Bullen CL, Liesegang TJ, McDonald TJ, et al. Ocular complications of Wegener's granulomatosis. Ophthalmology 1983;90:279-90.

13 Bacons PA, Kitas GD. Significance of vasculitis in RA. $\mathrm{Br}$ Ann Rheum Dis 1994;53:621-3.

14 Emery P, Salmon M. Early rheumatoid arthritis: time to aim for remission. Ann Rheum Dis 1995;54:944-7.

15 Hoffman GS, Leavitt RY, Fleisher TA, et al. Treatment of Wegener's granulomatosis with intermittent high-dose intravenous cyclophosphamide. Am f Med 1990;89:403 10.

16 Watts RA, Carruthers DM, Scott DG. Epidemiology of systemic vasculitis: changing incidence or definition? Sem Arthritis Rheum 1995;25:28-34. 